\title{
Questionnaire for Identification of Dyslexia Signs (QUESDI)
}

Alexandre Lucas de Araújo Barbosa ${ }^{12}$, Cíntia Alves Salgado-Azoni1 23

${ }^{1}$ Written Language, Interdisciplinarity and Learning Laboratory, Natal, RN, Brazil

${ }^{2}$ Psychology Department, Federal University of Rio Grande do Norte, Natal, RN, Brazil

${ }^{3}$ Speech-Language-Hearing Sciences Department, Federal University of Rio Grande do Norte, Natal, RN, Brazil

Correspondence:

Cíntia Alves Salgado-Azoni

Written Language, Interdisciplinarity and Learning Laboratory

Speech-Language-Hearing Sciences Department

Federal University of Rio Grande do Norte

R. Gen. Gustavo Cordeiro de Farias, s/n - Petrópolis, Natal - RN, 59012-570

cintiasalgadoazoni@gmail.com 


\section{Introduction}

Dyslexia is a specific learning disorder that affects word decoding, fluency, reading comprehension, and spelling (American Psychiatric Association, 2014). The early signs are visible throughout the school years, and it is possible to identify these signs through screening questionnaires. Early identification supports a better prognosis, through intervention in emergent reading skills (Cassidy and Cassidy, 2019).

In Brazil, the availability of screening instruments for dyslexia is scarce (Alves, Lima, Salgado-Azoni, Carvalho, Ciasca, 2015). Therefore, we built a screening instrument based on an integrative literature review, as well as based on the Phonological Deficit Theory (Caylak, 2010), named Questionnaire for Identification of Dyslexia Signs (QUESDI). QUESDI is suitable for parents and teachers of children between kindergarten and the third year of elementary school. It is available on the EduEdu application, from the ABCD Institute.

\section{Questionnaire items}

The questionnaire is composed of questions about oral language development (phonology, access to the lexicon, phonological awareness), knowledge of letters, spelling (grapheme-phoneme relationship, grammar of the Portuguese language), reading (speed, fluency, comprehension), phonological working memory, family history, reading habits and attention. All items took into account the signs of dyslexia, as well as those that can differentiate it from other diagnostic conditions, according to several scientific studies.

\section{Scoring and results analysis}

The instrument's score works as follows: rarely - 1 point; occasionally - 2 points; often - 3 points; always - 4 points. The final score will be based on the sum of all responses by the child's guardian or teacher. The instrument is still in the validation process, but cut-off scores were defined for each age group, considering the skills expected for each school grade. In 
the end, the child will be classified as low risk, medium risk, or high risk for dyslexia (Table

1).

Table 1. Cut-off scores and results analysis.

\begin{tabular}{|c|c|}
\hline \multicolumn{2}{|c|}{ Parents version - child education } \\
\hline & $\begin{array}{l}7 \text { to } 14 \text { points - low risk } \\
15 \text { to } 21 \text { points - medium risk } \\
22 \text { to } 28 \text { points - high risk }\end{array}$ \\
\hline \multicolumn{2}{|c|}{ Parents version - elementary school } \\
\hline First year & $\begin{array}{c}16 \text { to } 34 \text { points - low risk } \\
35 \text { to } 46 \text { points - medium risk } \\
47 \text { to } 64 \text { points - high risk }\end{array}$ \\
\hline Second year & $\begin{array}{l}16 \text { to } 32 \text { points - low risk } \\
33 \text { to } 40 \text { points - medium risk } \\
41 \text { to } 64 \text { points - high risk }\end{array}$ \\
\hline Third year & $\begin{array}{l}16 \text { to } 25 \text { points - low risk } \\
26 \text { to } 37 \text { points - medium risk } \\
38 \text { to } 64 \text { points - high risk }\end{array}$ \\
\hline \multicolumn{2}{|c|}{ Teachers version - child education } \\
\hline & $\begin{array}{l}6 \text { to } 13 \text { points - low risk } \\
14 \text { to } 20 \text { points - medium risk } \\
21 \text { to } 24 \text { points - high risk }\end{array}$ \\
\hline \multicolumn{2}{|c|}{ Teachers version - elementary school } \\
\hline First year & $\begin{array}{c}13 \text { to } 31 \text { points - low risk } \\
32 \text { to } 42 \text { points - medium risk } \\
43 \text { to } 52 \text { points - high risk }\end{array}$ \\
\hline Second year & $\begin{array}{l}13 \text { to } 28 \text { points - low risk } \\
29 \text { to } 38 \text { points - medium risk } \\
39 \text { to } 52 \text { points - high risk }\end{array}$ \\
\hline Third year & $\begin{array}{l}13 \text { to } 24 \text { points - low risk } \\
25 \text { to } 36 \text { points - medium risk } \\
37 \text { to } 52 \text { points - high risk }\end{array}$ \\
\hline
\end{tabular}




\section{References}

Alves, R.., Lima, R., Salgado-Azoni, C., Carvalho, C., \& Ciasca, S. (2015). Teste para Identificação de Sinais de Dislexia: processo de construção. Estudos de Psicologia (Campinas), 32(3), 383-393. https://doi.org/10.1590/0103-166x2015000300004

American Psychiatric Association. (2014). Manual diagnóstico e estatístico de transtornos mentais: DSM-5 (5th ed.) [Review of Manual diagnóstico e estatístico de transtornos mentais: DSM-5]. Artmed.

Cassidy, B., \& Cassidy, L. (2019). Early Screening and Intervention for Students with Dyslexia [Review of Early Screening and Intervention for Students with Dyslexia]. State Education Standard, 19(2), 28-32.

Caylak. (2010). The Studies about Phonological Deficit Theory in Children with Developmental Dyslexia: Review. American Journal of Neuroscience, 1(1), 1-12. https://doi.org/10.3844/ajnsp.2010.1.12 


\begin{tabular}{|c|c|}
\hline \multicolumn{2}{|l|}{ Oral language } \\
\hline $\begin{array}{l}\text { 1. My child still has speech disorders (e.g., replaces /v/ } \\
\text { for /f/, in the word "vaca", he/she speaks "faca"). }\end{array}$ & $\begin{array}{l}\text { ( ) Rarely } \\
\text { ( ) Occasionally } \\
\text { ( ) Often } \\
(\text { ) Always }\end{array}$ \\
\hline $\begin{array}{l}\text { 2. My child had speech disorders that were not expected } \\
\text { for his/her age (e.g., at four years of age, he/she } \\
\text { spoke "tota tola", instead of "coca cola"). }\end{array}$ & $\begin{array}{l}\text { ( ) Rarely } \\
\text { ( ) Occasionally } \\
\text { ( ) Often } \\
(\text { ) Always }\end{array}$ \\
\hline $\begin{array}{l}\text { 3. My child has difficulties learning and remembering } \\
\text { names. }\end{array}$ & $\begin{array}{l}\text { ( ) Rarely } \\
\text { ( ) Occasionally } \\
\text { ( ) Often } \\
(\text { ) Always }\end{array}$ \\
\hline 4. My child has difficulties with nursery rhymes. & $\begin{array}{l}\text { ( ) Rarely } \\
\text { ( ) Occasionally } \\
\text { ( ) Often } \\
\text { ( ) Always }\end{array}$ \\
\hline
\end{tabular}

\section{Letter knowledge}

5. My son recognizes and knows the name of the letters.

$\begin{array}{ll}( & \text { ) Rarely } \\ ( & \text { Occasionally } \\ ( & \text { Often } \\ ( & \text { ) Always }\end{array}$

\section{Family history}

6. There are people in my family who have/had difficulties with speech or reading.

$\begin{array}{ll}\text { ( } & \text { ) Rarely } \\ \text { ( } & \text { Occasionally } \\ \text { ( } & \text { Often } \\ \text { ( } & \text { ) Always }\end{array}$

\section{Reading habits}

7. My child is interested in children's books.

$$
\begin{array}{ll}
\text { ( ) Rarely } \\
\text { ( ) Occasionally } \\
\text { ( ) Often } \\
\text { ( ) Always }
\end{array}
$$


QUESDI - Parents version (elementary school)

\begin{tabular}{|c|c|}
\hline \multicolumn{2}{|l|}{ Oral language } \\
\hline $\begin{array}{l}\text { 1. My child still has speech disorders (e.g., replaces /v/ } \\
\text { for /f/, in the word "vaca", he/she speaks "faca"). }\end{array}$ & $\begin{array}{l}\text { ( ) Rarely } \\
\text { ( ) Occasionally } \\
\text { ( ) Often } \\
\text { ( ) Always }\end{array}$ \\
\hline $\begin{array}{l}\text { 8. My child had speech disorders that were not expected } \\
\text { for his/her age (e.g., at four years of age, he/she } \\
\text { spoke "tota tola", instead of "coca cola"). }\end{array}$ & $\begin{array}{l}\text { ( ) Rarely } \\
\text { ( ) Occasionally } \\
\text { ( ) Often } \\
\text { ( ) Always }\end{array}$ \\
\hline $\begin{array}{l}\text { 2. My child has difficulties learning and remembering } \\
\text { names. }\end{array}$ & $\begin{array}{l}\text { ( ) Rarely } \\
\text { ( ) Occasionally } \\
\text { ( ) Often } \\
\text { ( ) Always }\end{array}$ \\
\hline 3. My child has difficulties with nursery rhymes. & $\begin{array}{l}\text { ( ) Rarely } \\
\text { ( ) Occasionally } \\
\text { ( ) Often } \\
\text { ( ) Always }\end{array}$ \\
\hline
\end{tabular}

\section{Letter knowledge}

4. My child recognizes and knows the name of the ( ) Rarely letters.

( ) Occasionally

( ) Often

( ) Always

\section{Spelling}

5. My child replaces letters in writing. E.g.: when writing the word "vaca", he/she writes "faca".

My child reverses or omits letters in writing. E.g.: when writing the word "prato", he/she writes "parto" or "pato".

) Rarely
Occasionally
) Often
) Always
) Rarely
Occasionally
Often
) Always

\section{Reading}

9. My child has difficulties reading new words.

$$
\begin{aligned}
& \text { ( ) Rarely } \\
& \text { ( ) Occasionally } \\
& \text { ( ) Often } \\
& \text { ( ) Always }
\end{aligned}
$$




\begin{tabular}{|c|c|}
\hline $\begin{array}{l}\text { 10. My child has difficulties with rhythm, speed, and } \\
\text { intonation of reading. }\end{array}$ & $\begin{array}{l}\text { ( ) Rarely } \\
\text { ( ) Occasionally } \\
\text { ( ) Often } \\
(\text { ) Always }\end{array}$ \\
\hline $\begin{array}{l}\text { 11. When I compare my child's reading performance with } \\
\text { other children's performances, I see that other } \\
\text { children are reading much better than he/she is. }\end{array}$ & $\begin{array}{l}\text { ( ) Rarely } \\
(\text { ) Occasionally } \\
(\text { ) Often } \\
(\text { ) Always }\end{array}$ \\
\hline 12. My child has difficulties with reading comprehension. & $\begin{array}{ll}( & \text { ) Rarely } \\
( & \text { ) Occasionally } \\
( & \text { ) Often } \\
( & \text { ) Always }\end{array}$ \\
\hline
\end{tabular}

\begin{tabular}{|c|c|}
\hline \multicolumn{2}{|l|}{ Memory } \\
\hline $\begin{array}{l}\text { 13. My child easily forgets information that he/she just } \\
\text { read. }\end{array}$ & $\begin{array}{l}\text { ( ) Rarely } \\
\text { ( ) Occasionally } \\
\text { ( ) Often } \\
\text { ( ) Always }\end{array}$ \\
\hline $\begin{array}{l}\text { 14. My child easily forgets information that he/she just } \\
\text { heard. }\end{array}$ & $\begin{array}{l}\text { ( ) Rarely } \\
\text { ( ) Occasionally } \\
\text { ( ) Often } \\
\text { ( ) Always }\end{array}$ \\
\hline
\end{tabular}

\section{Family history}

15. There are people in my family who have/had ( ) Rarely difficulties with speech or reading.

( ) Occasionally

( ) Often

( ) Always

\section{Reading habits}

16. My child is interested in children's books.

( ) Rarely
( ) Occasionally
( ) Often
( $)$ Always

\section{Attention}

17. My child listens carefully to instructions for performing daily activities at home (e.g., asking to pick up an object and put it away).

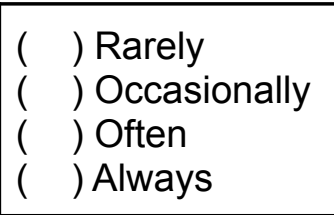




\begin{tabular}{|c|c|}
\hline \multicolumn{2}{|l|}{ Oral language } \\
\hline $\begin{array}{l}\text { 1. My student still has speech disorders (e.g., replaces } \\
\text { /v/ for /f/, in the word "vaca", he/she speaks "faca"). }\end{array}$ & $\begin{array}{l}\text { ( ) Rarely } \\
\text { ( ) Occasionally } \\
\text { ( ) Often } \\
(\text { ) Always }\end{array}$ \\
\hline $\begin{array}{l}\text { 2. My student had speech disorders that were not } \\
\text { expected for his/her age (e.g., at four years of age, } \\
\text { he/she spoke "tota tola", instead of "coca cola"). }\end{array}$ & $\begin{array}{l}\text { ( ) Rarely } \\
\text { ( ) Occasionally } \\
(\text { ) Often } \\
\text { ( ) Always }\end{array}$ \\
\hline 3. My student has difficulties with nursery rhymes. & $\begin{array}{l}\text { ( ) Rarely } \\
\text { ( ) Occasionally } \\
\text { ( ) Often } \\
(\text { ) Always }\end{array}$ \\
\hline
\end{tabular}

\title{
Letter knowledge
}

4. My student recognizes and knows the name of the letters.

$\begin{array}{ll}( & \text { ) Rarely } \\ ( & \text { Occasionally } \\ ( & \text { ) Often } \\ ( & \text { ) Always }\end{array}$

\section{Memory}

5. My student easily forgets information that he/she just heard.

$\begin{array}{ll}( & \text { ) Rarely } \\ \text { ( } & \text { ) Occasionally } \\ ( & \text { Often } \\ ( & \text { ) Always }\end{array}$

\section{Attention}

6. My student can perform activities when requested in the classroom, according to his/her level of development.

\author{
) Rarely \\ ) Occasionally \\ ) Often \\ ( ) Always
}


QUESDI - Teachers version (elementary school)

\begin{tabular}{|c|c|}
\hline \multicolumn{2}{|l|}{ Oral language } \\
\hline $\begin{array}{l}\text { 1. My student still has speech disorders (e.g., replaces } \\
\text { /v/ for /f/, in the word "vaca", he/she speaks "faca"). }\end{array}$ & $\begin{array}{l}\text { ( ) Rarely } \\
\text { ( ) Occasionally } \\
\text { ( ) Often } \\
(\text { ) Always }\end{array}$ \\
\hline $\begin{array}{l}\text { 2. My student had speech disorders that were not } \\
\text { expected for his/her age (e.g., at four years of age, } \\
\text { he/she spoke "tota tola", instead of "coca cola"). }\end{array}$ & $\begin{array}{l}\text { ( ) Rarely } \\
\text { ( ) Occasionally } \\
(\text { ) Often } \\
\text { ( ) Always }\end{array}$ \\
\hline 3. My student has difficulties with nursery rhymes. & $\begin{array}{l}\text { ( ) Rarely } \\
\text { ( ) Occasionally } \\
\text { ( ) Often } \\
(\text { ) Always }\end{array}$ \\
\hline
\end{tabular}

\section{Letter knowledge}

4. My student recognizes and knows the name of the letters.

$\begin{array}{ll}\text { ( } & \text { ) Rarely } \\ \text { ( }) \text { Occasionally } \\ \text { ( ) Often } \\ \text { ( }) \text { Always }\end{array}$

\section{Spelling}

5. My student replaces letters in writing. E.g.: when writing the word "vaca", he/she writes "faca".

) Rarely

) Occasionally

) Often

) Always

6. My student reverses or omits letters in writing. E.g.: when writing the word "prato", he/she writes "parto" or "pato".
( ) Rarely

) Occasionally

( ) Often

( ) Always

\begin{tabular}{|c|c|}
\hline \multicolumn{2}{|l|}{ Reading } \\
\hline 7. My student has difficulties reading new words. & $\begin{array}{l}\text { ( ) Rarely } \\
\text { ( ) Occasionally } \\
\text { ( ) Often } \\
\text { ( ) Always }\end{array}$ \\
\hline $\begin{array}{l}\text { 8. My child has difficulties with rhythm, speed, and } \\
\text { intonation of reading. }\end{array}$ & $\begin{array}{l}\text { ( ) Rarely } \\
\text { ( ) Occasionally } \\
\text { ( ) Often } \\
\text { ( ) Always }\end{array}$ \\
\hline
\end{tabular}




\begin{tabular}{|c|c|}
\hline $\begin{array}{l}\text { 9. When I compare my child's reading performance with } \\
\text { other children's performances, I see that other } \\
\text { children are reading much better than he/she is. }\end{array}$ & $\begin{array}{l}\text { ( ) Rarely } \\
\text { ( ) Occasionally } \\
\text { ( ) Often } \\
(\text { ) Always }\end{array}$ \\
\hline 10. My child has difficulties with reading comprehension. & $\begin{array}{l}\text { ( ) Rarely } \\
\text { ( ) Occasionally } \\
\text { ( ) Often } \\
\text { ( ) Always }\end{array}$ \\
\hline
\end{tabular}

\begin{tabular}{|c|c|}
\hline \multicolumn{2}{|l|}{ Memory } \\
\hline $\begin{array}{l}\text { 11. My student easily forgets information that he/she just } \\
\text { read. }\end{array}$ & $\begin{array}{ll}\text { ( } & \text { ) Rarely } \\
\text { ( ) Occasionally } \\
\text { ( ) Often } \\
\text { ( } \text { ) Always }\end{array}$ \\
\hline $\begin{array}{l}\text { 12. My student easily forgets information that he/she just } \\
\text { heard. }\end{array}$ & $\begin{array}{ll}\text { ( } & \text { ) Rarely } \\
\text { ( }) \text { Occasionally } \\
\text { ( ) Often } \\
\text { ( ) Always }\end{array}$ \\
\hline
\end{tabular}

\section{Attention}

13. My student is able to perform assignments when prompted for classroom activities (e.g., asking to pick up the math book and turn it to page 34).

$\begin{array}{ll}\text { ( } & \text { ) Rarely } \\ \text { ( } & \text { Occasionally } \\ \text { ( } & \text { Often } \\ \text { ( } & \text { ) Always }\end{array}$

